\title{
Chapter 3 \\ Camouflage Variations on a Theme of the Nymphalid Ground Plan
}

\author{
Takao K. Suzuki
}

\begin{abstract}
Lepidopteran camouflage patterns offer sophisticated and captivated examples of morphological evolution. Previous studies focused on how and why camouflage patterns are modulated at the microevolutionary level and determined, for instance, the adaptive role of camouflage patterns in avoiding predator attacks. However, less attention has been paid to the macroevolution of camouflage, including the evolutionary paths leading to the origination of leaf mimicry patterns. To understand the deep origins and evolvability of camouflage patterns, a key principle comes from a highly conserved ground plan (termed the nymphalid ground plan; NGP). The ground plan generates a variety of morphological forms, while it maintains its own type. This review introduces several seminal studies that used NGP-known features to reveal the macroevolutionary aspects of lepidopteran camouflage patterns, providing a roadmap for further understanding this biological phenomenon. The following core themes are discussed: (1) how complex camouflage patterns evolved (macroevolutionary pathways), (2) what kind of flexible mechanisms facilitate the origin of such complex patterns (macro-evolvability), and (3) how such complex patterns are tightly integrated through the coupling and uncoupling of ancestral developmental mechanisms (body plan character map). These approaches will provide new research lines for studying the evolution of camouflage patterns and the underlying flexibility of the NGP.
\end{abstract}

Keywords Crypsis and masquerade - Butterfly and moth - Comparative morphology - Macroevolution - Evolutionary path - Phylogenetic comparative methods • Tinkering • Morphological integration and modules • Morphometrics • Genotype-phenotype map

The original version of this chapter was revised. An erratum to this chapter can be found at https://doi.org/10.1007/978-981-10-4956-9_18

T.K. Suzuki (ه)

Transgenic Silkworm Research Unit, Division of Biotechnology, Institute of Agrobiological Sciences, National Agriculture and Food Research Organization (NARO), 1-2 Oowashi,

Tsukuba, Ibaraki 305-8634, Japan

e-mail: homaresuzuki@gmail.com 


\subsection{Introduction}

Complex and sophisticated camouflage patterns have fascinated many biologists (Poulton 1890; Cott 1940; Edmunds 1974; Ruxton et al. 2004; Stevens 2016). Recently, camouflage has been classified into two major types: crypsis (blended into environmental backgrounds to avoid detection by potential predators) and masquerade (special resemblance to natural objects to avoid recognition by potential predators) (Stevens and Merilaita 2009; Merilaita and Stevens 2011; Skelhorn et al. 2010a, b; Skelhorn 2015). Prominent cases of camouflage are found in butterfly and moth wing patterns, including tree bark crypsis in Biston betularia (van't Hof et al. 2016), lichen crypsis in Agriopodes fallax (Schmidt et al. 2014), leaf vein masquerade in the noctuid moth Oraesia excavata (Fig. 3.1a; Suzuki 2013) or in the nymphalid butterflies Kallima inachus and K. paralekta (Fig. 3.1b; Suzuki et al. 2014), and dried leaf masquerade in Polygonia c-album (Wiklund and Tullberg 2004). Most studies focused on the microevolutionary aspects of camouflage generation. For example, research on the industrial melanism shown in peppered moths deciphered both the adaptive significance (Cook et al. 2012) and the genetic basis of cryptic color variation (Cook and Saccheri 2013; van't Hof et al. 2016). Studies on the seasonal polyphenism of the butterflies Araschnia levana (Koch and Bückmann 1985), Bicyclus anynana (Brakefield and Larsen 1984; Monteiro et al. 2015), and Polygonia c-aureum (Fukada and Endo 1966; Endo 1984; Endo et al. 1988) have also uncovered hormonal switches in the generation of the cryptic patterns matching dry or autumnal color environments. In contrast, the macroevolution of camouflage has received little attention. The present review focuses on the comparative morphology of camouflage patterns in butterfly and moth wings and proposes a research roadmap for further advancing our understanding of the generative mechanisms underlying camouflage evolution.

For addressing the macroevolutionary aspects of lepidopteran camouflage, a key principle is that comparison of the anatomy of many species allows the extraction of

Fig. 3.1 Camouflage of moth and butterfly wing patterns. (a) Oraesia excavata. (b) Kallima inachus (Figure panel $\mathbf{a}$ is reproduced with modification from Suzuki (2013). Figure panel b is reproduced with modification from Suzuki et al. (2014)) a

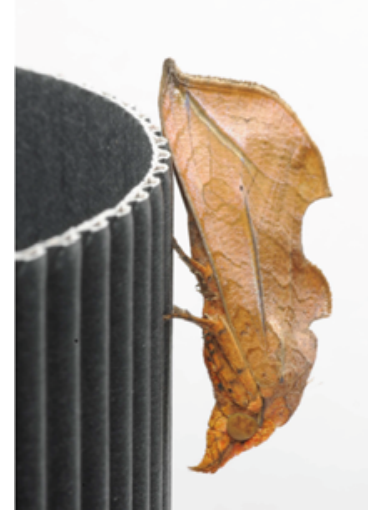

b

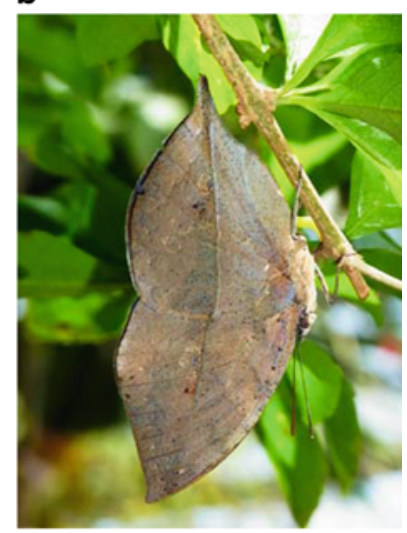


a common theme behind diversity, termed the "body plan" or "ground plan," which refers to the structural composition of organisms based on homologous elements shared among species (Wagner 2014). To date, butterfly and moth (at least within Macrolepidoptera) wing patterns are thought to be based on a highly conserved ground plan (termed the nymphalid ground plan, NGP; Fig. 3.2a; Schwanwitsch 1924; Süffert 1927; Nijhout 1991). The NGP describes the diversification of wing patterns as modifications of an assembly of discrete pattern elements shared among species (Schwanwitsch 1956; Nijhout 1991) and is suggested to be homologous and inherited across species. From the comparative morphology point of view, the essential question is how effective is the NGP scheme in understanding lepidopteran camouflage patterns? Moreover, if certain camouflage patterns are illustrated by the NGP, what information can this scheme provide for understanding
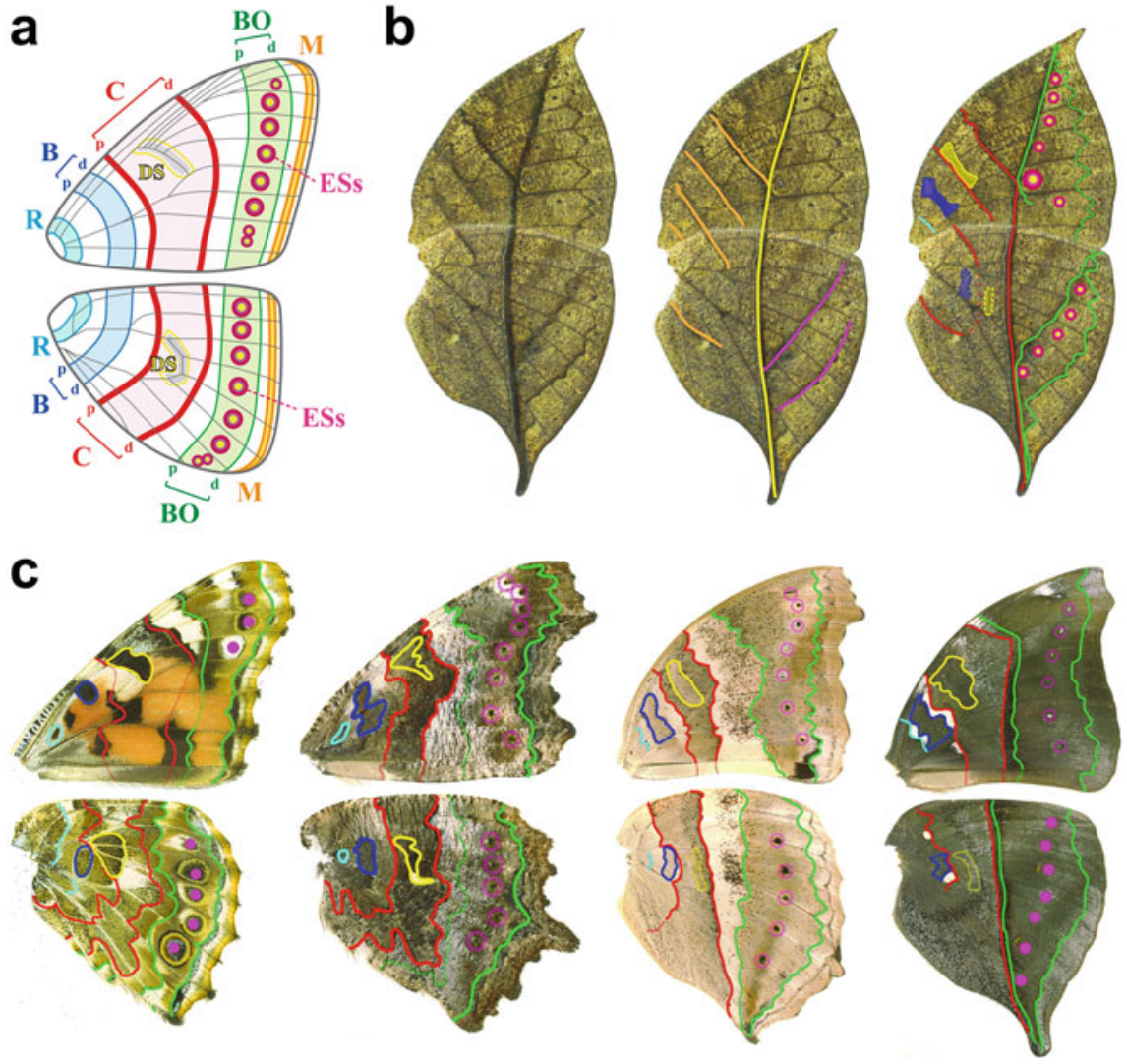

Fig. 3.2 Nymphalid ground plan and the variations generating diversified wing patterns. (a) Nymphalid ground plan (NGP). (b) Leaf vein-like pattern and the NGP of Kallima inachus. (c) NGP of Vanessa cardui, Nymphalis vaualbum, Yoma sabina, Doleschallia bisaltide (This figure is reproduced with modification from Suzuki et al. (2014)) 
lepidopteran camouflage patterns and can it contribute to the morphological evolution and organization of such spectacular examples of adaptation to the environment?

The present review introduces several NGP studies that are crucial for revealing the macroevolutionary aspects of lepidopteran camouflage patterns and provide a basis for further understanding this biological phenomenon. First, the foundations for using comparative morphology to identify homologous elements across species are described along with how NGP has led the way to the elaboration of diverse wing pattern configurations. Next, the potential of phylogenetic comparative methods to reveal the sequential evolutionary steps that built up leaflike patterns from nonmimetic ones is discussed. Third, the scheme of the NGP is used for discussing a flexible building logic of leaf mimicry patterns. Fourth, a methodological framework for analyzing the degree of integration and modularity in leaf vein-like pattern is proposed, and arguments favoring the evolutionary origin of $d e$ novo functional modules are presented. Finally, a research roadmap for further macroevolutionary studies on the origin and diversification of camouflage patterns is proposed.

\subsection{Morphological Foundations of the Nymphalid Ground Plan}

The concepts of body plan and ground plan are traditionally rooted in comparative morphology (Rieppel 1988). The criteria for identifying structural or positional homologs across different species were summarized by Remane (1952) and are considered a validated procedure in systematic and comparative morphology studies (Williams and Ebach 2008). These criteria consist of three principal rules: (1) similarity of topographical relationships, (2) similarity of special features, and (3) transformational continuity through intermediate ontogeny or phylogeny. The first criterion is logically consistent with Geoffroy St. Hilaire's "principe des connexions" (Saint-Hilaire 1818), the second is based on the specific properties of a character of interest, and the third is based on the evolutionary continuity of developmental genetic mechanisms underlying the character of interest. Although the concept of homology is still widely discussed (Patterson 1982; Roth 1988; Wagner 1989, 2007; Brower and Schawaroch 1996; Hall 2000), Remane's criteria remain valuable consensuses that crystallize empirical facts through numerous careful observations of morphological structures. Currently, these criteria provide a powerful tool to decipher the homology of anatomical structures in a broad spectrum of animals and plants (for animals: Nagashima et al. 2009; Hutchinson et al. 2011; Luo 2011; Holland et al. 2013; for plants: Sattler 1984; Buzgo et al. 2004).

The NGP is a scheme for describing homologous elements shared across species and thus should be evaluated within the logical framework of Remane's criteria. 
Although Remane's criteria were inherent to NGP studies by Schwanwitsch (1956) and Nijhout and Wray (1986), to my knowledge, there is no explicit citation to Remane's work in NGP studies. Recently, I tackled to apply Remane's criteria to analyze the NGP of Kallima inachus and $K$. paralekta leaf vein-like patterns and succeeded in demonstrating that these can be explained by the NGP (Fig. 3.2b; Suzuki et al. 2014), and the results were consistent with Schwanwitsch (1956) analysis and validated the empirical inference proposed by Süffert (1927). The wing patterns of species closely related to Kallima spp. can also be explained by the NGP, although these patterns differ from that found in Kallima spp. (Fig. 3.2c, only four species were selected; for further details, see Suzuki et al. 2014). Interestingly, these analyses revealed that the differences between the leaf vein-like pattern and the other non-leaf patterns resulted only from differences in the character states of NGP elements. Thus, comparative morphology provides in-depth information about the way of diversification of lepidopteran wing patterns, even in extreme cases such as leaf mimicry.

It is important to mention that the NGP framework has limitations, which are most evident when lepidopteran wing patterns have so dramatically deviated from a stereotypical pattern that they challenge reasonable homology assignments. For example, the wing patterns of some papilionids are intensively fragmented through dislocation and thus difficult to connect to the NGP (Mallet 1991). In the nymphalid butterflies Heliconius sp., the NGP has undergone complex rearrangements that culminated in a highly modified state (Mallet 1991), although NGP was previously reported for this genus (Nijhout and Wray 1988). In such cases, less derived species can provide clues on intermediate states and clarify the nature of homologous characters but are prone to misidentifications without a more mechanistic understanding of wing pattern architecture. To further understand the evolutionary trajectories of the NGP, it is necessary to investigate the molecular mechanisms underlying NGP. Previous studies revealed the molecular mechanisms underlying eyespots (ocelli), one of the NGP elements in butterfly wings (Carroll et al. 1994; Brakefield et al. 1996; Keys et al. 1999; Brunetti et al. 2001; Beldade and Brakefield 2002; Monteiro et al. 2006; Oliver et al. 2012; Monteiro et al. 2013; Monteiro 2015; Zhang and Reed 2016; Beldade and Peralta 2017). Molecular studies have also uncovered several morphogens (e.g., Wntl/wingless, WntA) and transcription factors (e.g., aristaless2, engrailed) associated with other elements of NGP (Brunetti et al. 2001; Monteiro et al. 2006; Martin and Reed 2010, 2014).

\subsection{Evolutionary Path: Gradual Evolutionary Steps Toward Leaf Vein-Like Patterns}

The ground plan architecture of lepidopteran wing patterns provides a starting point to investigate the evolutionary paths leading to complex camouflage patterns, but how can these trajectories be analyzed in exquisitely detailed phenotypes? 
Character polarity has been used in most studies investigating the evolutionary processes that generate traits (Donoghue 1989; Swofford and Maddison 1992; Wiley and Lieberman 2011), and it refers to the biased phylogenetic placement of certain states of a character of interest (Fig. 3.3a). Clear detection of character polarity indicates a nested hierarchical relationship between traits, whose character states are evolutionarily transformed from ancestral to derived states in a specific temporal order. As shown in Fig. 3.3a, the evolution of trait A follows that of the trait B. However, this approach has a crucial practical limitation: traits of interest often lack a clear character polarity. To cope with this limitation, some statistical methods, collectively termed phylogenetic comparative methods (PCMs), were developed for analyzing traits' evolution (Fig. 3.3b; Harvey and Pagel 1991; Losos and Miles 1994; Garamszegi 2014). In PCMs, statistical testing is incorporated into the examination of phylogenetic information and character states to analyze the evolution of traits (Pagel 1999a). Accordingly, these methods can be used to detect subtle nuances of trait evolution that lack a clear signature of character polarity and thus can be applied in a broad spectrum of scenarios featuring a complex distribution of character states. In such scenarios, PCMs can be used in the reconstruction of traits' ancestral states (Schluter et al. 1997; Pagel 1999b; Pagel et al. 2004) or to infer the temporal order in which traits evolved, within a phylogenetic framework (Pagel 1994; Pagel and Meade 2006).

a

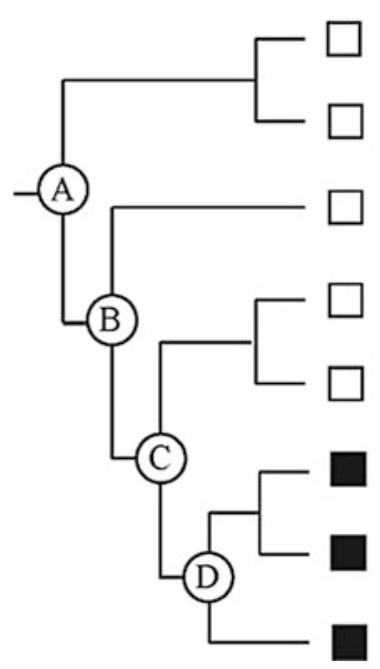

b

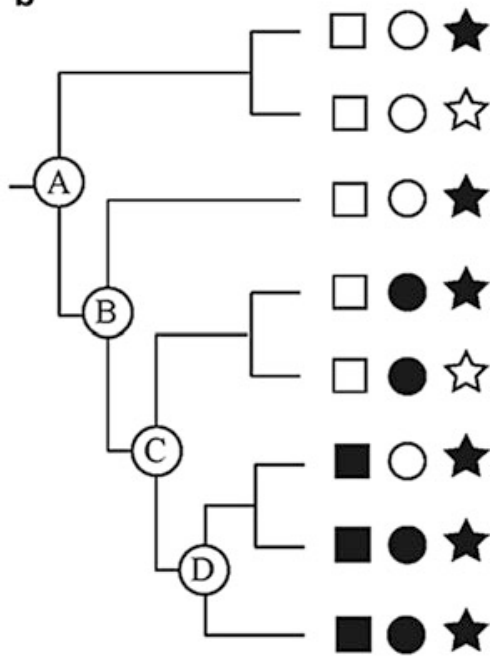

Fig. 3.3 How to infer macroevolutionary paths toward complex traits. (a) Simple case of character polarity, in which a trait (square) evolved from state 0 (open square) to state 1 (close square) at the node D of the phylogeny. (b) Complex case of character polarity, in which phylogenetic comparative methods were used to estimate the ancestral states of the traits (squares, circles, and stars) 
a

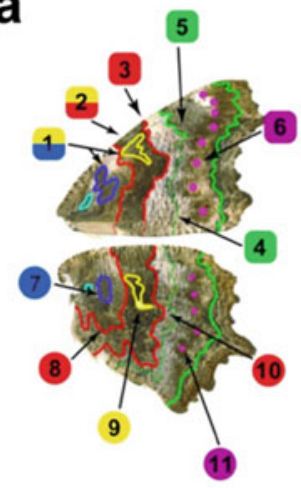

C

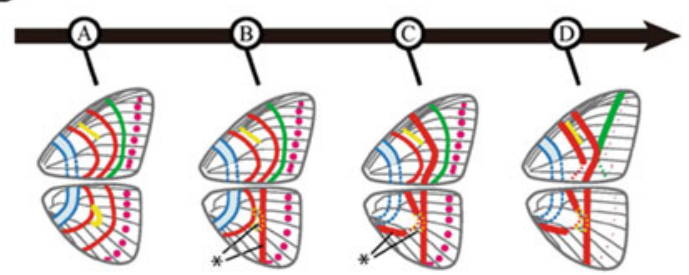

b

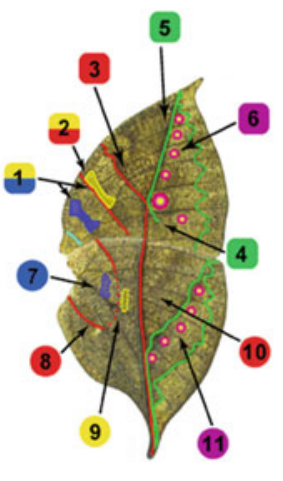

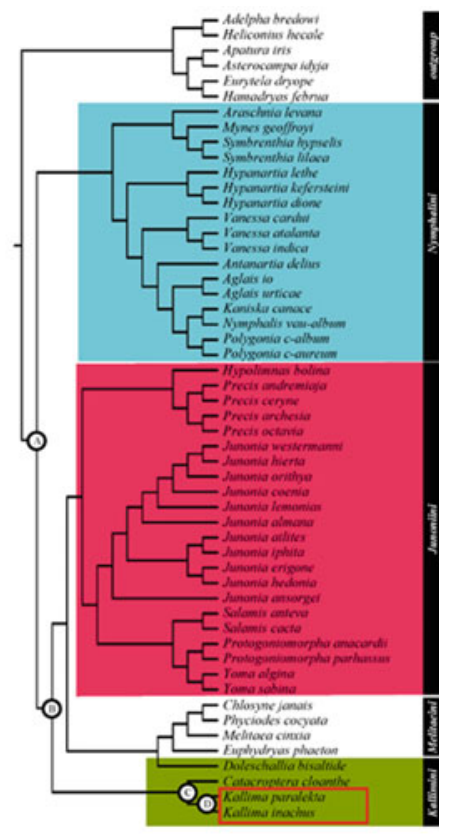

Fig. 3.4 Evolutionary steps that generated Kallima sp. leaf vein-like patterns. (a) Decomposition of wing patterns into 11 character states. (b, c) Reconstructed ancestral character states are represented at four selected nodes $(A, B, C$, and $D)$, which are illustrated as the time required for the evolutionary transformation of wing patterns (from $A$ to $D$ ). In the molecular phylogeny, the genus Kallima is evidenced using a red box (This figure is reproduced with modification from Suzuki et al. (2014))

The evolution of leaf resemblance in Kallima spp. has been a long-term conundrum and remains unresolved. Under a gradualistic view (Darwin 1871; Wallace 1889; Poulton 1890; Weissman 1902; Watson et al. 1936), the leaf mimicry pattern is a product of slow gradual evolution, with natural selection progressively perfecting masquerade forms; under the alternative saltationist view, leaf mimicry pattern evolved via relatively sudden leaps in the morphospace without intermediate forms (Mivart St 1871; Goldschmidt 1945). Despite the enthusiastic debate, no formal assessment of the tempo and mode of evolution in leaf mimicking has been provided so far. Recently, I applied PCMs to gain insight on the evolutionary paths that led to the leaf vein-like pattern in Kallima spp. (Fig. 3.4; Suzuki et al. 2014). If overall phenotypes are treated as integrated units, PCM analyses cannot reconstruct the evolutionary history of complex traits, simply by informing how many times the traits evolved (e.g., Mugleston et al. 2013). To avoid this, the butterfly wing patterns including the leaf patterns were decomposed into a set of several subcomponents using the NGP (Fig. 3.4a), which allowed inferring the ancestral states of each component and reconstructing the evolutionary process as the sum of the changes occurring in all 
components (Suzuki 2017). Thus, tracing ancestral states at various phylogenetic nodes illustrates the sequential transformation of the character states of multiple components that led to the complex traits (Fig. 3.4b). This analysis revealed the successive steps in the evolution of leaf masquerade patterns from nonmimetic wing patterns within a phylogenetic framework (Fig. 3.4c; Suzuki et al. 2014) and provided the first evidence for gradual evolutionary origin of leaf mimicry (Skelhorn 2015). Thus, combining NGP and PCMs information provides an insight into the structural complexity of lepidopteran wing patterns and the possibility to depict the evolutionary paths leading to the formation of complex and detailed patterns (Suzuki 2017).

\subsection{Tinkering: The Flexible Building Logic of Leaf Vein-Like Patterns}

In addition to the reconstruction of evolutionary paths described above, identifying the NGP of lepidopteran wing patterns will provide resources to assess the different ways to produce leaf vein-like patterns. Regarding this issue, Schwanwitsch (1956) described the NGP of several species presenting leaf patterns such as Siderone marthesia (Fig. 3.5a), Zaretis isidora (Fig. 3.5b), and K. inachus (Fig. 3.2b). According to his scheme, the mode of derivation from the NGP is in most part repeated in these three species. Interestingly, the genera Siderone and Zaretis (Charaxinae, a subfamily of Nymphalidae) are taxonomically distant from the genus Kallima (Nymphalinae), which is also supported by Wahlberg et al. (2009) molecular phylogeny. Because convergence is considered to represent independently evolved features that are both structurally and superficially similar (Stayton
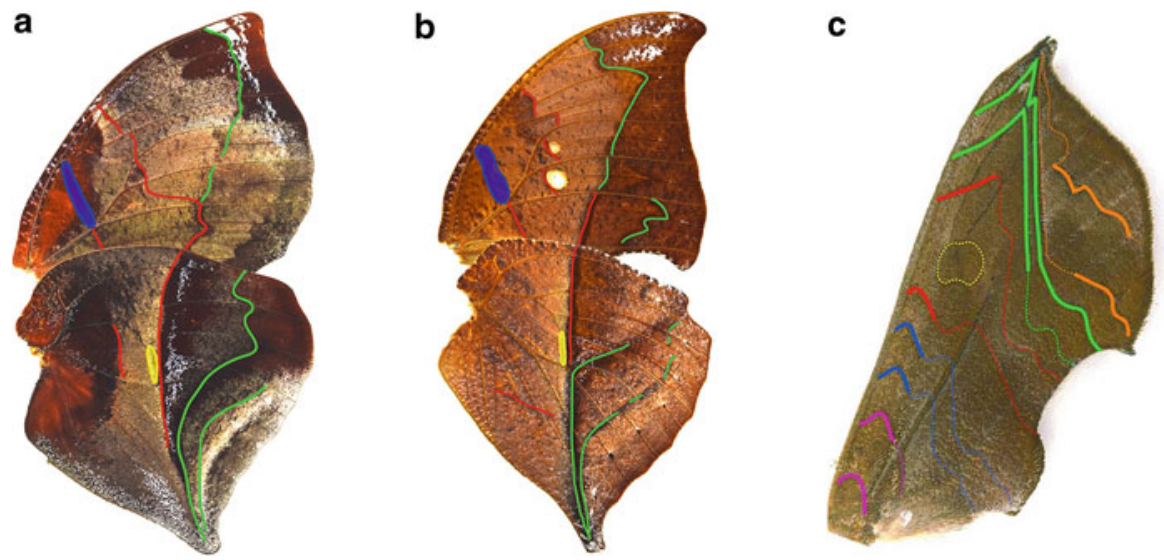

Fig. 3.5 Leaf vein-like variations on the same NGP theme. (a) Siderone marthesia. (b) Zaretis isidora. (c) Oraesia excavata. The NGP of S. marthesia and Z. isidora is based on Schwanwitsch (1956) (Figure panel c is reproduced with modification from Suzuki (2013)) 
2015), the similar mode of derivation from the NGP found in Charaxinae and Nymphalinae probably resulted from independent events of convergent evolution.

Does this similar mode of NGP-derived patterns, which seems to indicate that leaf pattern construction modes are quite constrained in butterflies, hold true for more distantly related taxa than Nymphalinae and Charaxinae? To address this question, I here compare the NGP of the leaf vein-like pattern found in the noctuid moth O. excavata, one of the most abundant moths in Northeast Asia (Fig. 3.1a), to that of $K$. inachus. Although these leaf vein-like patterns look similar, both consisting of a main vein and two sets of lateral veins, the way in which these two leaf patterns were built from the NGP is quite different (Figs. 3.2b and 3.5c). For example, in $K$. inachus butterflies, the main vein of the leaf pattern is derived from a green element (the proximal band of the border symmetry system) and a red element (the distal band of the central symmetry system), whereas in O. excavata, the main leaf vein is derived only from green elements (the border symmetry system). These observations showed that Lepidoptera leaf patterns can evolve through different paths, revealing a higher flexibility than that suggested from the analysis centered on nymphalid butterflies only.

This flexibility in leaf pattern building could be discussed within the concept of tinkering, which was in biology proposed by François Jacob (1977). This concept was described as "a tinkerer who does not know exactly what he is going to produce, but uses whatever he finds around him, whether it be pieces of string, fragments of wood, or old cardboards; in short it works like a tinkerer who uses everything at his disposal to produce some kind of workable object." Based on this statement, the leaf patterns of Kallima spp. and Oraesia spp. evolved in a tinkering mode of innovation, managing with odds and ends. Additionally, and although it might seem unexpected, the dead leaves of Charaxinae might have achieved the same construction style observed in Kallima as a result of tinkering evolution. Strictly speaking, tinkering likely refers to the evolutionary process of building up traits and not just to the traits. Thus, the flexible building logic of Lepidoptera leaf patterns might reflect the tinkering logic of the evolutionary processes behind them.

\subsection{Modularity: Developmental Modules of the NGP and a Simple Cryptic Pattern}

How a morphological structure is integrated is crucial to understand the genetic and developmental architecture of trait adaptation (Olson and Miller 1958; Cheverud 1996; Klingenberg 2008). The concept of morphological integration postulates that functionally related elements are tightly coupled (Olson and Miller 1958; Cheverud 1996). A special form of integration is modularity, in which units are tightly coupled but can be individually decoupled (Wagner and Altenberg 1996). Modularity results from the regulatory interactions of developmental mechanisms (Klingenberg 2008) and/or from accumulated structural changes shaped by natural 
a

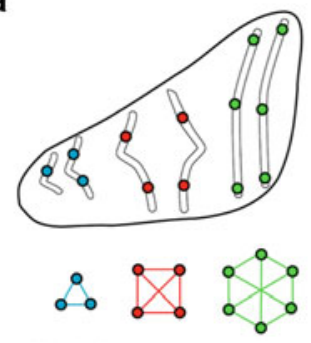

Modularity to common homologues

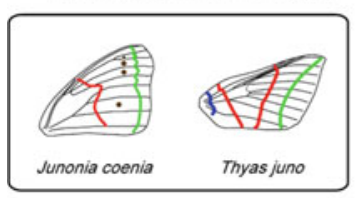

b

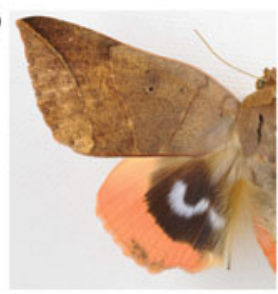

C

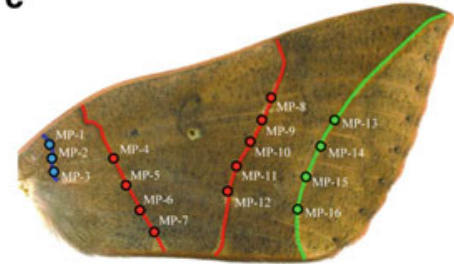

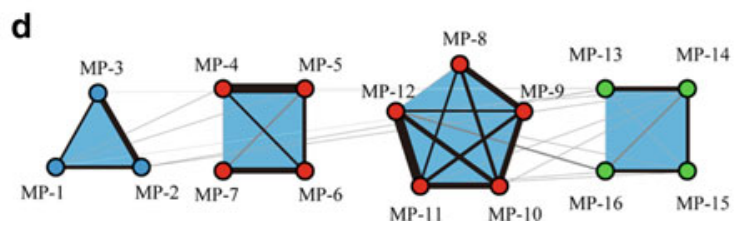

Fig. 3.6 Modularity of the simple cryptic pattern of Thyas juno. (a) Schematic illustration of divergence strategy in moth and butterfly wing patterns. The modularity of simple patterns corresponds to the original developmental modules of the NGP. (b) Forewings and hind wings of $T$. juno. (c) Forewings comprise four elements, each corresponding to an NGP element. (d) Morphological correlation network of the $T$. juno forewing pattern. In this correlation network, nodes represent measurement points and lines represent the correlations between measurement points (larger correlation coefficients are indicated by thicker arrow edges and darker lines). The modules detected are illustrated as light-blue areas (This figure is reproduced with modification from Suzuki (2013))

selection (Lande 1979; Arnold 1983; Wagner and Altenberg 1996). Previous studies suggested that the NGP is the sum of several developmental modules where each NGP element is genetically and/or developmentally autonomous (Fig. 3.6a; Nijhout 1991, 1994, 2001; Beldade and Brakefield 2002). In fact, the central symmetry system of the NGP appears to be a genetically and phenotypically independent unit (Brakefield 1984; Paulsen and Nijhout 1993; Paulsen 1994, 1996), and eyespots are developmental units formed by factors diffused from foci (Nijhout 1980; French and Brakefield 1995). These considerations strongly suggest that butterfly and moth wing patterns, including camouflage patterns, obey to NGP's rule of modularity.

How are lepidopteran camouflage patterns integrated and modularized? To address this issue, the relatively simple camouflage pattern of the noctuid moth Thyas juno was examined (Fig. 3.6b; Suzuki 2013). At rest, this species displays only the cryptic forewings covering the conspicuous hind wings, but, once it detects a potential enemy, the forewings are unfolded and display the warning-colored hind wings. The forewing pattern consists of four elements, each corresponding to an NGP element (Fig. 3.6c). To detect the modules involved in an overall wing pattern, I developed a new analytical method (termed morphological correlation network), which allows analyzing geometric morphometric data by combining graph theory and the statistical physics of spin glass (Suzuki 2013; Esteve-Altava 2016). This approach revealed that the modules involved in $T$. juno wing pattern corresponded to individual NGP symmetry elements, which might reflect the original modular 
architecture of the NGP (Fig. 3.6d) as supported by previous considerations regarding NGP organization (Nijhout 1991, 1994, 2001; Beldade and Brakefield 2002). Although studying a practical case is limited, at least in relatively simple camouflage patterns, these results supported the hypothesis that the genetic and developmental architectures underlying camouflage patterns reflect the original developmental modules of the NGP (Fig. 3.6a).

\subsection{Evolutionary Origin of De Novo Modules: Rewiring of the NGP Developmental Modules to Generate Functional Modules}

How modules of morphological structures originated is an important question to understand the complex adaptation of phenotypes (Wagner et al. 2007; Klingenberg 2008). A previous conceptual study proposed that modules evolved through the opposite processes of integration (coupling) and parcellation (uncoupling) (Wagner and Altenberg 1996). This conceptual framework seems to be crucial to comprehend the evolution of butterfly and moth wing patterns through modifications of the NGP. Contrasting to the early establishment of the conceptual basis, how de novo modules originated still remains poorly understood (Moczek et al. 2015). The question here is how modules of complex camouflage patterns originated within the context of morphological integration and parcellation.

To address this question, the modular architecture of the leaf vein-like pattern of O. excavata (Figs. 3.1a and 3.5c) was investigated using the morphological correlation network method (Suzuki 2013). This study revealed that the leaf pattern of $O$. excavata is highly modularized, with each module corresponding to each component of the leaf vein, implying the functional modules (Fig. 3.7b). To examine the extent of the association between these functional modules and the developmental modules of the NGP, the morphological correlation network of the O. excavata wing pattern was replotted (Fig. 3.7c). Interestingly, functional modules were generated by the coupling and uncoupling of NGP developmental modules. For example, the functional module of the left lateral vein (i.e., module 2) originated from coupling two distinct modules of the central and border symmetry systems, and the developmental module of the border symmetry system was uncoupled into three functional modules (i.e., modules 2, 3,4). Thus, this analysis clearly demonstrated that, at least in the evolution of complex camouflage patterns such as leaf masquerade, de novo modules originated through the reintegration of NGP developmental modules (Fig. 3.7a).

Unlike the previous studies in which the NGP was considered to comprise autonomous units (Fig. 3.6; Nijhout 1991, 1994, 2001; Beldade and Brakefield 2002), the modules in the O. excavata leaf pattern originated through reintegration to new modules (Fig. 3.7). This discrepancy could be due to differences between simple and complex patterns (Figs. 3.6a and 3.7a). Previous studies often 

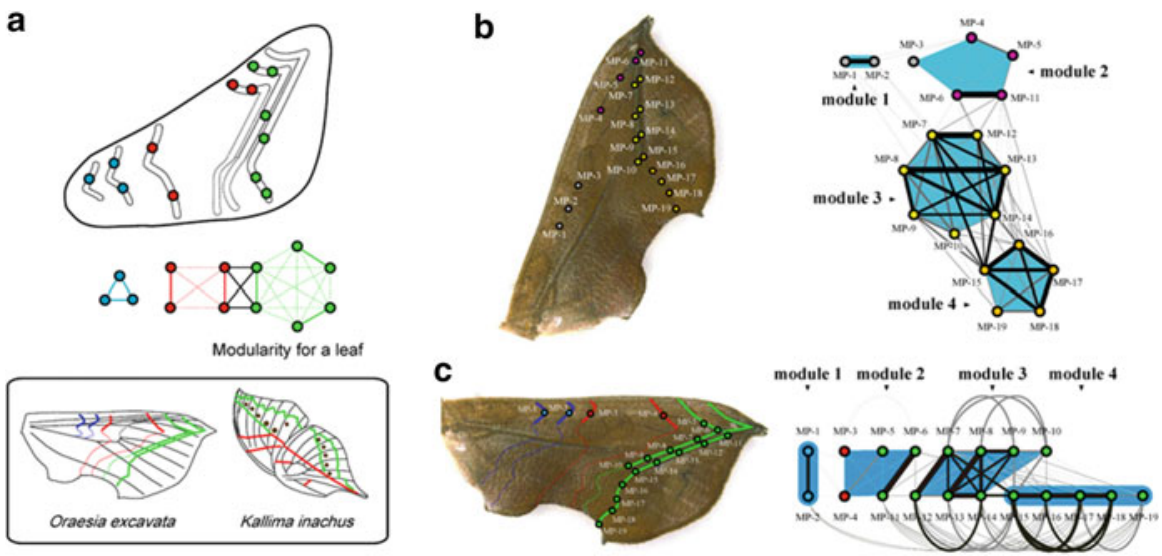

Fig. 3.7 Modularity of the leaf vein-like pattern of Oraesia excavata. (a) Schematic illustration of divergence strategy in moth and butterfly wing patterns. The modularity of complex patterns evolved through rewiring the original developmental modules of the NGP. (b) Forewings of O. excavata and its morphological correlation network. In this correlation network, nodes represent measurement points and lines represent correlations between measurement points (larger correlation coefficients are indicated by thicker arrow edges and darker lines). The modules detected are illustrated as lightblue areas. (c) Replot of the correlation network of O. excavata wing pattern based on the NGP (This figure was reproduced with modification from Suzuki (2013))

emphasized that the genetically and developmentally autonomous units of the NGP allowed further uncoupling pattern elements (e.g., dislocation), and such individualization is thought to allow establishing separate evolutionary trajectories, thereby contributing to the evolvability of lepidopteran wing patterns. In addition to this previous perspective, the present review emphasizes the importance of coupling of pattern elements in wing morphological diversification and proposes a new organizing principle, a "rewiring" strategy (i.e., coupling and uncoupling) of the NGP, in which a combination of decoupling and coupling processes "rewires" the correlations among common parts (Fig. 3.7a; Suzuki 2013).

\subsection{Next Research Programs}

Quantitative analyses, together with the scheme of the NGP, have begun to set a new path for understanding camouflage patterns of butterfly and moth wings. The NGP provides a foundation for the evolutionary pathways, evolvability, and genetic/developmental architecture underlying the complex and diversified camouflage patterns, through which the ground plan is modified. In this final section, further research programs are discussed. 


\subsubsection{Macroevolutionary Pathways Toward Camouflage Patterns}

Diversification based on NGP modifications is not a random process but occurs in a certain sequential order. As shown above, mathematical methods using Bayesian statistics enabled analyzing the evolutionary origin and sequential steps toward the various camouflage patterns (Suzuki et al. 2014; Suzuki 2017). This approach allows to test whether camouflage patterns originated gradually or suddenly and to analyze the evolutionary process through which modifications were accumulated generating camouflage patterns.

Furthermore, comparing multiple evolution processes allows examining evolutionary pathways considering whether processes within them are possible or not. For example, comparing the evolutionary processes involved in butterfly leaf masquerade and lichen cryptic patterns may reveal common/different evolutionary mechanisms between the different camouflage patterns. Similarly, comparing the evolutionary processes of leaf masquerade among distinct taxa may reveal how many pathways are involved in the evolution of lepidopteran leaf patterns and/or addressing the mechanisms allowing the multiple origins of leaf mimicry in Lepidoptera. To date, studies considering macroevolution discussed only the tempo, mode, and trends of evolution (Simpson 1944; Carroll 2001). In addition to these research directions, studying the evolutionary processes and pathways involved in complex and diversified traits is expected to add a new direction in the research field of macroevolution.

\subsubsection{Macro-evolvability of the NGP}

The deep involvement between body plan and evolvability has often been discussed (Vermeij 1973; Riedl 1978; Kirschner and Gerhart 1998; Graham et al. 2000). Regarding evolvability, Vermeij (1973) proposed the concept of versatility, which focuses on the number and range of independent parameters controlling morphological form. As described above, the evolution of the O. excavata leaf pattern involved the reintegration of the original developmental modules of the NGP (Fig. 3.7), suggesting that the increase in the number of parameters controlling shape allowed new adaptations, reflecting the versatility of the NGP (Suzuki 2013). In addition, the flexible logic of leaf mimicry patterns suggests a new component (e.g. tinkering) in the evolvability of the ground plan (Fig. 3.2b and 3.5). It has been pointed out that evolvability has various definitions, and Pigliucci proposed its classification in an evolutionary time scale (Pigliucci 2008). Following his definition, I would like to propose the term "macro-evolvability" to define the long time scale evolution that generates various forms through modifications of the ground plan.

Furthermore, one extreme case when examining the macro-evolvability of the ground plan is to determine under which circumstances the ground plan is partially or fully broken. In other words, this approach provides an insight into evolvable limitation of the NGP. Unlike that considered before Darwin's theory of evolution, 
the ground plan is also subject to natural selection, and therefore some or all of it might be broken with the evolutionary emergence of a specific form derived from the ground plan. Are there possibilities that the NGP was broken? The wing pattern of a mimicry butterfly, Heliconius sp., might be considered (Jiggins et al. 2017) a possible example of such a situation. Under this consideration, several questions are raised: How was the NGP deconstructed in Heliconius butterflies? What kind of natural selection promotes NGP loss? Does the evolutionary acquisition of Müllerian mimicry affect the loss of the NGP? To address such questions, it will be necessary to combine morphological and molecular studies to verify NGP integrity (Martin et al. 2012; Martin and Reed 2014), because the NGP might be difficult to identify in these butterflies.

\subsubsection{Body plan Character Map: Genetic and Developmental Architectures of the NGP}

What kind of genetic and developmental architectures underlies the ground plan? In previous studies, this issue was discussed from various perspectives, including the perspectives of transcriptomics (Duboule 1994; Kalinka et al. 2010; Irie and Kuratani 2011, 2014; Quint et al. 2012; Levin et al. 2016) and gene regulatory networks (Davidson and Erwin 2006; Wagner 2007). From the morphological integration and modularity perspective, two major schemes were proposed: the genotype-phenotype map (G-P map; Fig. 3.8a; Wagner and Altenberg 1996) and developmental mapping (D map; Fig. 3.8b; Klingenberg 2008). Both schemes describe how modules of traits were generated through internal interactions, but while the G-P map is based on genetics, the D map is based on ecological

a

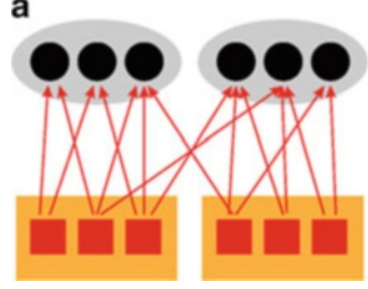

b

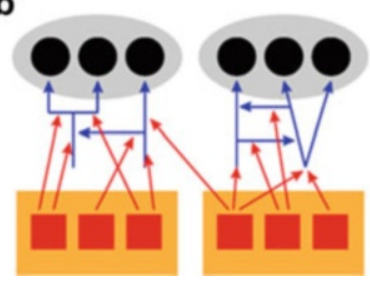

c

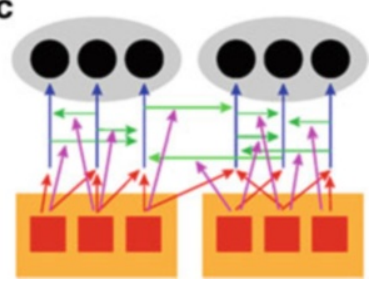

Fig. 3.8 Genetic and developmental architectures of a modularized phenotypic trait. (a) Genotype-phenotype map (G-P map). (b) Developmental map (D map). (c) Body plan character map (BC map). All schemes describe the relationship between genes (red squares) and the subcomponents (black circles) of a phenotypic trait, when the trait is modularized (gray circles). The G-P map describes the construction of modularity through changes in pleiotropic effects (red arrows), whereas the D map describes the modulated pathways of the developmental system (blue arrows) affected by changes in pleiotropy. The BC map describes the construction of modularity through the coupling (green arrows) and uncoupling (light green arrows) of the original developmental pathways of the ground plan (blue arrows), where subcomponents (black circles) are homologous parts, and each phenotypic trait is the ground plan of interest (Figure panel a was modified from Wagner and Altenberg (1996), and panel b was modified from Klingenberg (2008)) 
evolutionary developmental biology. These two schemes cover a broad spectrum of biological traits but are less likely to be practical for deciphering a specific genetic and developmental architecture of traits. From the perspective of comparative morphology, a specific scheme to comprehend the complexity and diversification of traits needs to be established.

How can the genetic and developmental architectures that create various forms by modification of the ground plan be depicted? Considering the experimental facts explained above, two major components seem to be involved: one arises from the original developmental modularity of the ground plan and the other from rewiring the developmental modules of the ground plan. In general, the ground plan is a sum of homologous parts, and it is thought that each homologous part constitutes one developmental module because each part is individually identifiable (Wagner 1989, 2014). An example of the component derived from rewiring the developmental modules of the NGP is the functional modules found in $O$. excavata leaf pattern (Suzuki 2013). In the present review, I propose a scheme for integrating the genetic and developmental architecture underlying the variations of a theme of the ground plan, termed Body plan Character Map (BC Map; Fig. 3.8c). This scheme describes the core generation process of the ground plan and the reorganization process that transforms it into various designs, which can only be revealed using the morphological approach described in this study combined with molecular data.

Acknowledgments I thank Toshio Sekimura, Fred Nijhout, and the Chubu University (Japan) for organizing the 2016 conference and stimulating the writing of this review. I would also like to thank Professor Hideki Sezutsu for his valuable comments on this manuscript and Professor Shigeru Kuratani for his inspired comments on comparative morphology discussion and importance of the macroevolutionary views. This manuscript was rigorously reviewed by two referees, Shigeyuki Koshikawa and Arnaud Martin.

\section{References}

Arnold SJ (1983) Morphology, performance and fitness. Am Zool 23:347-361

Beldade P, Brakefield PM (2002) The genetics and evo-devo of butterfly wing patterns. Nat Rev Genet 3:442-452

Beldade P, Peralta CM (2017) Developmental and evolutionary mechanisms shaping butterfly eyespots. Curr Opin Insect Sci 19:22-29

Brakefield PM (1984) The ecological genetics of quantitative characters of Maniola jurtina and other butterflies. In: Vane-Wright RI, Ackery PR (eds) Symposia of the royal entomological society, the biology of butterflies. Academic Press, London, pp 167-190

Brakefield PM, Larsen TB (1984) The evolutionary significance of dry and wet season forms in tropical butterflies. Biol J Linn Soc 22:1-22

Brakefield PM, Gates J, Keys D, Kesbeke F, Wijngaarden PJ, Monteiro A, French V, Carroll SB (1996) Development, plasticity and evolution of butterfly eyespot patterns. Nature 384:236-242

Brower AVZ, Schawaroch V (1996) Three steps of homology assessment. Cladistics 12:265-272

Brunetti CR, Selegue JE, Monteiro A, French V, Brakefield PM, Carroll SB (2001) The generation and diversification of butterfly eyespot color patterns. Curr Biol 11:1578-1585 
Buzgo M, Soltis DE, Soltis PS, Ma H (2004) Towards a comprehensive integration of morphological and genetic studies of floral development. Trends Plant Sci 9:164-173

Carroll SB (2001) Chance and necessity: the evolution of morphological complexity and diversity. Nature 409:1102-1109

Carroll SB, Gates J, Keys D, Paddock SW, Panganiban GF, Selegue JE, Williams JA (1994) Pattern formation and eyespot determination in butterfly wings. Science 265:109-114

Cheverud JM (1996) Developmental integration and the evolution of pleiotropy. Am Zool $36: 44-50$

Cook LM, Saccheri IJ (2013) The peppered moth and industrial melanism: evolution of a natural selection case study. Heredity 110:207-212

Cook LM, Grant BS, Saccheri IJ, Mallet J (2012) Selective bird predation on the peppered moth: the last experiment of Michael Majerus. Biol Lett. doi:10.1098/rsbl.2011.1136

Cott HB (1940) Adaptive coloration in animals. Methuen and Co., London

Darwin C (1871) The descent of man. John Murray, London

Davidson EH, Erwin DH (2006) Gene regulatory networks and the evolution of animal body plans. Science 311:796-800

Donoghue MJ (1989) Phylogenies and the analysis of evolutionary sequences, with examples from seed plants. Evolution 43:1137-1156

Duboule D (1994) Temporal collinearity and the phylotypic progression: a basis for the stability of a vertebrate Bauplan and the evolution of morphologies through heterochrony. Development (Suppl):135-142

Edmunds M (1974) Defence in animals. Longman, New York

Endo K (1984) Neuroendocrine regulation of the development of seasonal forms of the Asian comma butterfly Polygonia c-aureum. Dev Growth Diff 26:217-222

Endo K, Masaki T, Kumagai K (1988) Neuroendocrine regulation of the development of seasonal morphs in the Asian comma butterfly, Polygonia c-aureum L. difference in activity of summermorph-producing hormone from brain extracts of the long-day and short-day pupae. Zool Sci 5:145-152

Esteve-Altava B (2016) In search of morphological modules: a systematic review. Biol Rev. doi:10.1111/brv.12284

French V, Brakefield PM (1995) Eyespot development of butterfly wings: the focal signal. Dev Biol 168:112-123

Fukada S, Endo K (1966) Hormonal control of the development of seasonal forms in the butterfly Polygonia c-aureum L. Proc Jpn Acad 42:1082-1987

Garamszegi LZ (ed) (2014) Modern phylogenetic comparative methods and their application in evolutionary biology, concepts and practice. Springer, Heidelberg

Goldschmidt RB (1945) Mimetic polymorphism, a controversial chapter of Darwin. Q Rev Biol 20:205-230

Graham LE, Cook ME, Busse JS (2000) The origin of plants: body plan changes contributing to a major evolutionary radiation. Proc Natl Acad Sci U S A 97:4535-4540

Hall B (ed) (2000) Homology: the hierarchical basis of comparative biology. Academic Press, San Diego

Harvey PH, Pagel M (1991) The comparative method in evolutionary biology. Oxford University Press, Oxford

Holland LZ, Carvalho JE, Escriva H, Laudet V, Schubert M, Shimeld SM, Yu J-K (2013) Evolution of bilaterian central nervous systems: a single origin? EvoDevo 4:27

Hutchinson JR, Delmer C, Miller CE, Hildebrandt T, Pitsillides AA, Boyde A (2011) From flat foot to fat foot: structure, ontogeny, function, and evolution of elephant "sixth toes.". Science 334:1699-1703

Irie N, Kuratani S (2011) Comparative transcriptome analysis reveals vertebrate phylotypic period during organogenesis. Nat Commun 2. doi:10.1038/ncomms 1248

Irie N, Kuratani S (2014) The developmental hourglass model: a predictor of the basic body plan? Development 141:4649-4655 
Jacob F (1977) Evolution and tinkering. Science 196:1161-1166

Jiggins CD, Wallbank RWR, Hanly JJ (2017) Waiting in the wings: what can we learn about gene co-option from the diversification of butterfly wing patterns? Phil Trans R Soc B 372:20150485. doi:10.1098/rstb.2015.0485

Kalinka AT, Varga KM, Gerrard DT, Preibisch S, Corcoran DL, Jarrells J, Ohler U, Bergman CM, Tomancak P (2010) Gene expression divergence recapitulates the developmental hourglass model. Nature 468:811-814

Keys DN, Lewis DL, Selegue JE, Pearson BJ, Goodrich LV, Johnson RL, Gates J, Scott MP, Carroll SB (1999) Recruitment of a hedgehog regulatory circuit in butterfly eyespot evolution. Science 283:532-534

Kirschner M, Gerhart J (1998) Evolvability. Proc Natl Acad Sci U S A 95:8420-8427

Klingenberg CP (2008) Morphological integration and developmental modularity. Ann Rev Ecol Evol Syst 39:115-132

Koch PB, Bückmann D (1985) The seasonal dimorphism of Araschnia levana L. (Nymphalidae) in relation to hormonal controlled development. Verb Dt Zool Ges 78:260

Lande R (1979) Quantitative genetic analysis of multivariate evolution, applied to brain: body size allometry. Evolution 33:402-416

Levin M, Anavy L, Cole AG, Winter E, Mostov N, Khair S, Senderovich N, Kovalev E, Silver DH, Feder M, Fernandez-Valverde SL, Nakanishi N, Simmons D, Simakov O, Larsson T, Liu S-Y, Jerafi-Vider A, Yaniv K, Ryan JF, Martindale MQ, Rink JC, Arendt D, Degnan SM, Degnan BM, Hashimshony T, Yanai I (2016) The mid-developmental transition and the evolution of animal body plans. Nature 531:637-641

Losos JB, Miles DB (1994) Adaptation, constraint, and the comparative method: phylogenetic issues and methods. In: Wainwright PC, Reilly S (eds) Ecological morphology: integrative organismal biology. University of Chicago Press, Chicago, pp 60-98

Luo Z-X (2011) Developmental patterns in Mesozoic evolution of mammal ears. Ann Rev Ecol Evol Syst 42:355-380

Mallet J (1991) Variations on a theme? Nature 354:368

Martin A, Reed RD (2010) Wingless and aristaless2 define a developmental ground plan for moth and butterfly wing pattern evolution. Mol Biol Evol 27:2864-2878

Martin A, Reed RD (2014) Wnt signaling underlies evolution and development of the butterfly wing pattern symmetry systems. Dev Biol 395:367-378

Martin A, Papa R, Nadeau NJ, Hill RI, Counterman BA, Halder G, Jiggins CD, Kronforst MR, Long AD, McMillan WO, Reed RD (2012) Diversification of complex butterfly wing patterns by repeated regulatory evolution of a Wnt ligand. Proc Natl Acad Sci U S A 109:12632-12637

Merilaita S, Stevens M (2011) Crypsis through background matching. In: Stevens M, Merilaita S (eds) Animal camouflage, mechanisms and function. Cambridge University Press, Cambridge, pp 17-33

Mivart St GJ (1871) On the genesis of species. Macmillan, London

Moczek AP, Sears KE, Stollewerk A, Wittkopp PJ, Diggle P, Dworkin I, Ledon-Rettig C, Matus DQ, Roth S, Abouheif E, Brown FD, Chiu C-H, Cohen CS, Tomaso AWD, Gilbert SF, Hall B, Love AC, Lyons DC, Sanger TJ, Smith J, Specht C, Vallejo-Marin M, Extavour CG (2015) The significance and scope of evolutionary developmental biology: a vision for the 21 st century. Evol Dev 17:198-219

Monteiro A (2015) Origin, development, and evolution of butterfly eyespots. Annu Rev Entomol 60:253-271

Monteiro A, Glaser G, Stockslager S, Glansdorp N, Ramos D (2006) Comparative insights into questions of lepidopteran wing pattern homology. BMC Dev Biol 6:52

Monteiro A, Chen B, Ramos DM, Oliver JC, Tong X, Guo M, Wang W-K, Fazzino L, Kamal F (2013) Distal-less regulates eyespot patterns and melanization in Bicyclus butterflies. J Exp Zool B 320:321-331 
Monteiro A, Tong X, Bear A, Liew SF, Bhardwaj S, Wasik BR, Dinwiddie A, Bastianelli C, Cheong WF, Wenk MR, Cao H, Prudic KL (2015) Differential expression of ecdysone receptor leads to variation in phenotypic plasticity across serial homologs. PLoS Genet 11:e1005529

Mugleston JD, Song H, Whiting MF (2013) A century of paraphyly: a molecular phylogeny of katydids (Orthoptera: Tettigoniidae) supports multiple origins of leaf-like wings. Mol Phylo Evol 69:1120-1134

Nagashima H, Sugahara F, Takechi M, Ericsson R, Kawashima-Ohya Y, Narita Y, Kuratani S (2009) Evolution of the turtle body plan by the folding and creation of new muscle connections. Science 325:193-196

Nijhout HF (1980) Pattern formation on lepidopteran wings: determination of an eyespot. Dev Biol 80:267-274

Nijhout HF (1991) The development and evolution of butterfly wing patterns. Smithsonian Institution Press, Washington, DC

Nijhout HF (1994) Symmetry systems and compartments in lepidopteran wings: the evolution of a patterning mechanism. Development (Suppl):225-233

Nijhout HF (2001) Elements of butterfly wing patterns. J Exp Zool 291:213-295

Nijhout HF, Wray GA (1986) Homologies in the colour patterns of the genus Charaxes (Lepidoptera: Nymphalidae). Biol J Linn Soc 28:387-410

Nijhout HF, Wray GA (1988) Homologies in the colour patterns of the genus Heliconius (Lepidoptera: Nymphalidae). Linn Soc Biol J 33:345-365

Oliver JC, Tong XL, Gall LF, Piel WH, Monteiro A (2012) A single origin for nymphalid butterfly eyespots followed by widespread loss of associated gene expression. PLoS Genet 8:e1002893

Olson EC, Miller RL (1958) Morphological integration. University of Chicago Press, Chicago

Pagel M (1994) Detecting correlated evolution on phylogenies: a general method for the comparative analysis of discrete characters. Proc R Soc B 255:37-45

Pagel M (1999a) Inferring the historical patterns of biological evolution. Nature 401:877-884

Pagel M (1999b) The maximum likelihood approach to reconstructing ancestral character states of discrete characters on phylogenies. Syst Biol 48:612-622

Pagel M, Meade A (2006) Bayesian analysis of correlated evolution of discrete characters by reversible-jump Markov chain Monte Carlo. Am Nat 167:808-825

Pagel M, Meade A, Barker D (2004) Bayesian estimation of ancestral character states on phylogenies. Syst Biol 53:673-684

Patterson C (1982) Morphological characters and homology. In: Joysey KA, Friday AE (eds) Problems of phylogenetic reconstruction. Academic Press, London, pp 21-74

Paulsen SM (1994) Quantitative genetics of butterfly wing color patterns. Dev Genet 15:79-91

Paulsen SM (1996) Quantitative genetics of the wing color pattern in the buckeye butterfly (Precis coenia and Precis evarete): evidence against the constancy of g. Evolution 50:1585-1597

Paulsen SM, Nijhout HF (1993) Phenotypic correlation structure among elements of the color pattern in Precis coenia (Lepidoptera: Nymphalidae). Evolution 47:593-618

Pigliucci M (2008) Is evolvability evolvable? Nat Rev Genet 9:75-82

Poulton EB (1890) The colours of animals: their meaning and use, especially considered in the case of insects. Kegan Paul, Trench, Trübner and Co., Ltd., London

Quint M, Drost H-G, Gabel A, Ullrich KK, Bönn U, Grosse I (2012) A transcriptomic hourglass in plant embryogenesis. Nature 490:98-101

Remane A (1952) Die Grundlagen des Naturlichen Systems, der Vergleichenden Anatomie und der Phylogenetik. Theoretische Morphologie und Systematik I. Geest \& Portig K.-G., Leipzig

Riedl R (1978) Order in living organisms: a systems analysis of evolution. Wiley, New York

Rieppel O (1988) Fundamentals of comparative biology. Birkhauser Verlag, Basel

Roth VL (1988) The biological basis of homology. In: Humphries CJ (ed) Ontogeny and systematics. Columbia University Press, New York, pp 1-26

Ruxton GD, Sherratt TN, Speed MP (2004) Avoiding attack: the evolutionary ecology of Crypsis, warning signals and mimicry. Oxford University Press, Oxford

Saint-Hilaire EG (1818) Philosophie Anatomique, Tome Premiere. J. B. Baillière, Paris 
Sattler R (1984) Homology-a continuing challenge. Syst Botany 9:382-394

Schluter D, Price T, Mooers AØ, Ludwig D (1997) Likelihood of ancestor states in adaptive radiation. Evolution 51:1699-1711

Schmidt BC, Wagner DL, Zacharczenko BV, Zahiri R, Anweiler GG (2014) Polyphyly of lichencryptic dagger moths: synonymy of Agriopodes Hampson and description of a new basal acronictine genus, Chloronycta, gen. n. (Lepidoptera, Noctuidae). Zookeys 421:115-137

Schwanwitsch BN (1924) On the ground-plan of wing-pattern in Nymphalids and certain other families of the Rhopalocerous Lepidoptera. Proc Zool Soc Lond B 34:509-528

Schwanwitsch BN (1956) Color-pattern in Lepidoptera. Entomologeskoe Obozrenie 35:530-546

Simpson GG (1944) Tempo and mode in evolution. Columbia University Press, New York

Skelhorn J (2015) Masquerade. Curr Biol 25:R643-R644

Skelhorn J, Rowland HM, Ruxton GD (2010a) The evolution and ecology of masquerade. Biol J Linn Soc 99:1-8

Skelhorn J, Rowland HM, Speed MP, Ruxton GD (2010b) Masquerade: camouflage without crypsis. Science $327: 51$

Stayton CT (2015) The definition, recognition, and interpretation of convergent evolution, and two new measures for quantifying and assessing the significance of convergence. Evolution 69:2140-2153

Stevens M (2016) Cheats and deceits: how animals and plants exploit and mislead. Oxford University Press, Oxford

Stevens M, Merilaita S (2009) Animal camouflage: current issues and new perspectives. Phil Trans R Soc B 364:423-427

Süffert F (1927) Zur vergleichenden analyse der schmetterlingszeichnumg. Biol Zentralblatt 47:385-413

Suzuki TK (2013) Modularity of a leaf moth-wing pattern and a versatile characteristic of the wing-pattern ground plan. BMC Evol Biol 13:158

Suzuki TK (2017) On the origin of complex adaptive traits: progress since the Darwin vs. Mivart debate. J Exp Zool B 328:304-320

Suzuki TK, Tomita S, Sezutsu H (2014) Gradual and contingent evolutionary emergence of leaf mimicry in butterfly wings. BMC Evol Biol 14:229

Swofford DL, Maddison WP (1992) Parsimony, character-state reconstructions, and evolutionary inferences. In: Mayden RL (ed) Systematics, historical ecology, \& north american freshwater fishes. Stanford University Press, Palo Alto, pp 186-223

van't Hof AE, Campagne P, Rigden DJ, Yung CJ, Lingley J, Quail MA, Hall N, Darby AC, Saccheri IJ (2016) The industrial melanism mutation in British peppered moths is a transposable element. Nature 534:102-105

Vermeij GJ (1973) Adaptation, versatility and evolution. Syst Zool 22:466-477

Wagner GP (1989) The biological homology concept. Ann Rev Ecol Syst 20:51-69

Wagner GP (2007) The developmental genetics of homology. Nat Rev Genet 8:473-479

Wagner GP (2014) Homology, genes, and evolutionary innovation. Princeton University Press, Princeton

Wagner GP, Altenberg L (1996) Complex adaptations and the evolution of evolvability. Evolution 50:967-976

Wagner GP, Pavlicev M, Cheverud JM (2007) The road to modularity. Nat Rev Genet 8:921-931

Wahlberg N, Leneveu J, Kodandaramaiah U, Peña C, Nylin S, Freitas AVL, Brower AVZ (2009) Nymphalid butterflies diversify following near demise at the cretaceous/tertiary boundary. Proc R Soc B 276:4295-4302

Wallace AR (1889) Darwinism: an exploitation of the theory of natural selection with some of its applications. MacMillan \& Co., London

Watson DMS, Timofeeff-Ressovsky NW, Salisbury EJ, Turrill WB, Jenkin TJ, Ruggles Gates R, Fisher RA, Diver C, Hale Carpenter GD, Haldane JBS, MacBrid EW, Salaman RN (1936) A discussion on the present state of the theory of natural selection. Proc R Soc B 121:43-73

Weissman A (1902) The evolution theory. Edward Arnold, London 
Wiklund C, Tullberg BS (2004) Seasonal polyphenism and leaf mimicry in the comma butterfly. Anim Behav 68:621-627

Wiley EO, Lieberman BS (2011) Phylogenetics: theory and practice of phylogenetic systematics, 2nd edn. Wiley, Hoboken

Williams DM, Ebach MC (2008) Foundations of systematics and biogeography. Springer, New York

Zhang L, Reed RD (2016) Genome editing in butterflies reveals that spalt promotes and distal-less represses eyespot colour patterns. Nat Commun 7:11769

Open Access This chapter is licensed under the terms of the Creative Commons Attribution 4.0 International License (http://creativecommons.org/licenses/by/4.0/), which permits use, sharing, adaptation, distribution and reproduction in any medium or format, as long as you give appropriate credit to the original author(s) and the source, provide a link to the Creative Commons license and indicate if changes were made.

The images or other third party material in this chapter are included in the chapter's Creative Commons license, unless indicated otherwise in a credit line to the material. If material is not included in the chapter's Creative Commons license and your intended use is not permitted by statutory regulation or exceeds the permitted use, you will need to obtain permission directly from the copyright holder.

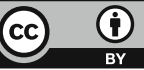

